\title{
A New Method for Specific Emitter Identification With Results on Real Radar Measurements
}

\author{
Gokhan Gok $^{(}$, Yasar Kemal Alp, and Orhan Arikan ${ }^{\circledR}$, Member, IEEE
}

\begin{abstract}
Specific Emitter Identification (SEI) is the process of specifically identifying mobile transmitters by extracting unique features from the precise measurements of their emitted signals. A novel signal processing scheme with two stages is proposed for the identification of specific radar emitters. In the first stage, the received radar pulses are accurately time aligned and coherently integrated in order to increase the Signal-toNoise (SNR) ratio. Using this technique, measurements with SNR improvements of more than $25 \mathrm{~dB}$ are obtained, enabling detection of subtle differences between different emitters. In the second step, Variational Mode Decomposition (VMD) is used to decompose both the envelope and the instantaneous frequency of the received signal into a set of modes. Then, these mod signals are characterized by using a group of features for identification. We demonstrate highly successful identification performance with the proposed method on real radar datasets.
\end{abstract}

Index Terms-Radar specific emitter identification, unintentional modulation on pulse, variational mode decomposition, classification, time-frequency domain features.

\section{INTRODUCTION}

$\mathbf{I}^{\mathrm{T}}$ $\mathrm{N}$ conventional Electronic Warfare (EW), radar signals are classified using classical parameters or features such as the Pulse Width (PW), Pulse Repetition Interval (PRI), Radio Frequency (RF), Antenna Scan Type/Period (AST/ASP) and the Intentional Modulation on Pulse (IMOP). However, classification of radars using conventional techniques becomes a more and more challenging task in modern EW systems, as the number of emitters and their sophistication in a typical EW scenario grows significantly. Moreover, advances in digital technology allow designers to implement different countermeasures in order to degrade detection and classification performance of EW systems. Hence, detailed intra-pulse analysis of the received Intermediate Frequency (IF) signals becomes even more critical [1].

Radar Specific Emitter Identification (SEI) is an important Electronic Intelligence (ELINT) activity that aims to recognize individual emitters using features related to the so-called

Manuscript received August 7, 2019; revised December 11, 2019 and March 16, 2020; accepted April 9, 2020. Date of publication April 17, 2020; date of current version May 5, 2020. The associate editor coordinating the review of this manuscript and approving it for publication was Dr. Matthieu R. Bloch. (Corresponding author: Gokhan Gok.)

Gokhan Gok is with the Radar, Electronic Warfare and Intelligence Systems Division, ASELSAN Inc., 06830 Ankara, Turkey, and also with the Department of Electrical and Electronics Engineering, Bilkent University, 06800 Ankara, Turkey (e-mail: ggok@ee.bilkent.edu.tr).

Yasar Kemal Alp is with the Radar, Electronic Warfare and Intelligence Systems Division, ASELSAN Inc., 06830 Ankara, Turkey.

Orhan Arikan is with the Department of Electrical and Electronics Engineering, Bilkent University, 06800 Ankara, Turkey.

Digital Object Identifier 10.1109/TIFS.2020.2988558
Unintentional Modulation On Pulse (UMOP) [2]. This goal is accomplished by precisely measuring the unintentional modulations on radar signals due to manufacturing differences of the transmitter hardware, including the power amplifiers and radar casing. In other words, UMOP is like a fingerprint of an emitter and can be used to even identify transmitters from the same manufacturing line.

In the literature, there are several proposed techniques for extracting radar fingerprints. In [3] and [4], the spectra of intercepted radar signals are used to generate features for the fingerprint analysis. In [5], non linear effects of power amplifiers are modelled and analyzed for the SEI. In [6], a complete SEI architecture is proposed where features are generated using higher order cumulants of radar signals and then k-Nearest Neighbor ( $\mathrm{kNN}$ ) classifier is then used to assign emitters to specific classes. In [7], basic SEI features related to characteristics of a square wave including the rise time, fall time and overshoot are discussed and, results for a dataset consisting of two real radar signals are given. However, the details of feature extraction are not presented. Another approach employing Empirical Model Decomposition (EMD) [8] is introduced in [9]. Although the mod signals shown for two different emitters indicate variations, no further processing leading to automatic classification is presented. A common issue in the literature on radar SEI is the very limited use of real field data if not at all. Moreover, due to concerns of confidentiality, some critical steps of the proposed techniques are left out.

As stated in [2], SEI is not limited to identification of radar emitters. For example, in [10], effects related to oscillator phase noise of wireless devices are analyzed and used for classification. In [11]-[15], unintentional modulations related to the power amplifiers of communication systems are modelled using methods such as the Taylor series expansion, and results of the proposed algorithms are demonstrated in different communication scenarios and channels by using synthetically generated data. A new approach where the Hilbert spectrum of the received signal is fed into a deep residual network is introduced in [16] and results on single-hop and relaying communication scenarios are presented. Another machine learning based approach where the compressed bispectrum of the measured data is fed into a convolutional neural network is proposed in [17]. Yet again, these studies also lack results with real field data or do not provide any proof about the validity of the data generation models.

In this work, we propose a novel and complete scheme for radar SEI. Preliminary results were presented in [18]. Here, 
we introduce a detailed description and extensive test results with real field data. The proposed method consists of two main stages. In the first stage, preprocessing of the acquired radar pulses is performed to reveal underlying unintentional modulations, which are very subtle for state of the art radar emitters of the same kind. For these emitters, pulse shapes of transmitters are usually very similar and differences could only be observed in a high Signal-to-Noise Ratio (SNR) regime that is not common in most practical EW scenarios. As a result, we propose a preprocessing scheme to integrate the pulses emitted by the same radar with the same carrier or radio frequency (RF). For successful integration, it is crucial to align collected pulses accurately in time and compensate for their phase offsets. In the proposed method, the collected samples of the acquired pulses are modelled as the discrete samples of a piece-wise continuous function [19]. Then, by choosing a reference pulse (typically the one with the highest SNR), the relative time delays between the reference pulse and all the remaining ones, which are far below the sampling interval of the receiver, are estimated by solving an optimization problem. Note that, since the pulses are modelled as a continuous function, the estimated time alignment delays are on a continuum rather than a fixed discrete grid, i.e., the estimation resolution is beyond the limit set by the sampling frequency of the Electronic Support Measures (ESM) receiver. The time aligned pulses are then coherently integrated to obtain a single pulse with improved SNR. In the second stage, SEI features are obtained by using Variational Mode Decomposition (VMD) [20]. The proposed feature extraction technique decomposes the envelope and instantaneous frequency of the radar pulses into components that have compact support in the frequency domain. A set of features characterizing these compact signals is then obtained and used for identification.

This paper is organized as follows. In Sec. II, a detailed formulation of the pulse alignment technique is given. In Sec. III, we present the results obtained by applying the pulse alignment technique on both synthetically generated and real radar signals. In Sec. IV, the VMD based feature extraction method is introduced. In Sec. V, a computational complexity analysis of the proposed technique is given. In Sec. VI, the performance of the proposed technique is shown on a dataset that consists of real radar signals. The manuscript is concluded in Sec. VII.

\section{Time Alignment of Pulses}

Let $g_{p}(t), 1 \leq p \leq P$, represent $P$ pulse signals emitted by the same radar with the same carrier frequency. The complex Inphase-Quadrature (IQ) samples of the $p^{\text {th }}$ pulse measured by a digital ESM receiver with sampling rate $F_{S}$ can be written as:

$$
g_{p}\left(t_{n}\right)=a_{p}\left(t_{n}\right) e^{j \phi_{p}\left(t_{n}\right)}+z_{p}(n), 1 \leq n \leq N_{p},
$$

where $t_{n}$ is the sampling instance of the $n^{\text {th }}$ sample, $N_{p}$ is the number of collected samples, $z_{p}(n)$ is a sample of circularly symmetric complex white Gaussian noise with standard deviation $\sigma_{z}$. Here, $\phi_{p}\left(t_{n}\right)$ and $a_{p}\left(t_{n}\right)$ represent the instantaneous phase and the envelope of the pulse, respectively. For illustration, the envelopes and instantaneous frequencies of $P=383$ real radar pulses are shown in Fig. 1. Note that the
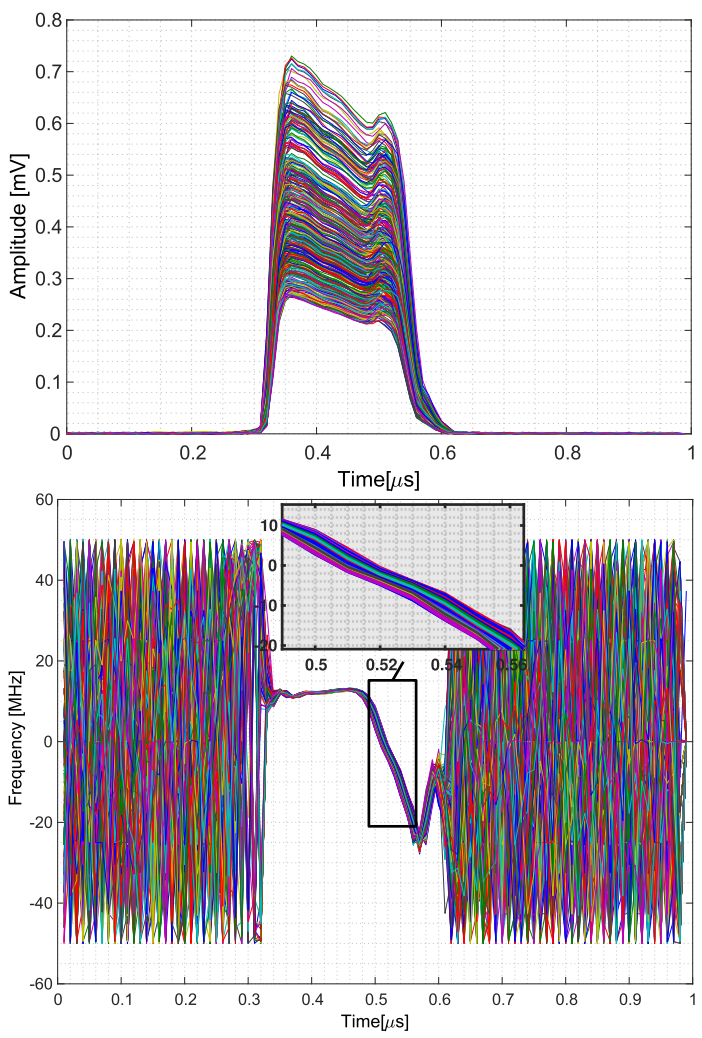

Fig. 1. Envelopes (above) and instantaneous frequencies (below) of 383 real radar pulses.

instantaneous frequency can be approximated as:

$$
f_{p}\left(t_{n}\right)=\left(\phi_{p}\left(t_{n}\right)-\phi_{p}\left(t_{n-1}\right)\right) F_{s} /(2 \pi) .
$$

As observed in Fig. 1 although these pulses are roughly aligned by the digital receiver according to their times of arrival (TOA), their instantaneous frequency curves appear to spread along the frequency axis at each $t_{n}$, which indicates imprecision in their alignment.

Consider the following model for each pulse:

$$
g_{p}\left(t_{n}\right)=c_{p} g_{r}\left(t_{n}-\tau_{p}\right)+z_{p}(n) .
$$

Here $g_{r}\left(t_{n}\right)$ is the reference pulse for the time alignment and amplitude/phase-offset correction procedures that will be implemented on the remaining pulses; $\tau_{p}$ is the time delay, and $c_{p}$ is the complex coefficient controlling the amplitude/phaseoffset. After estimating the $c_{p}$ and $\tau_{p}$ parameters for each pulse, the integrated pulse with higher SNR can be formed as:

$$
\hat{g}\left(t_{n}\right)=\frac{1}{P} \sum_{p=1}^{P} g_{p}\left(t_{n}+\hat{\tau}_{p}\right) e^{-j L \hat{c}_{p}},
$$

where $\angle($.) is the operator which returns the phase of its argument in radians. If all the $c_{p}$ and $\tau_{p}$ parameters are estimated correctly, the standard deviation of the noise in the integrated pulse will be $\sigma_{z} / \sqrt{P}$. As a result, we can bound the effective SNR after the integration operation as:

$$
\mathrm{SNR}_{\min }+10 \log _{10}(P) \leq \mathrm{SNR}_{\mathrm{eff}} \leq \mathrm{SNR}_{\max }+10 \log _{10}(P),
$$



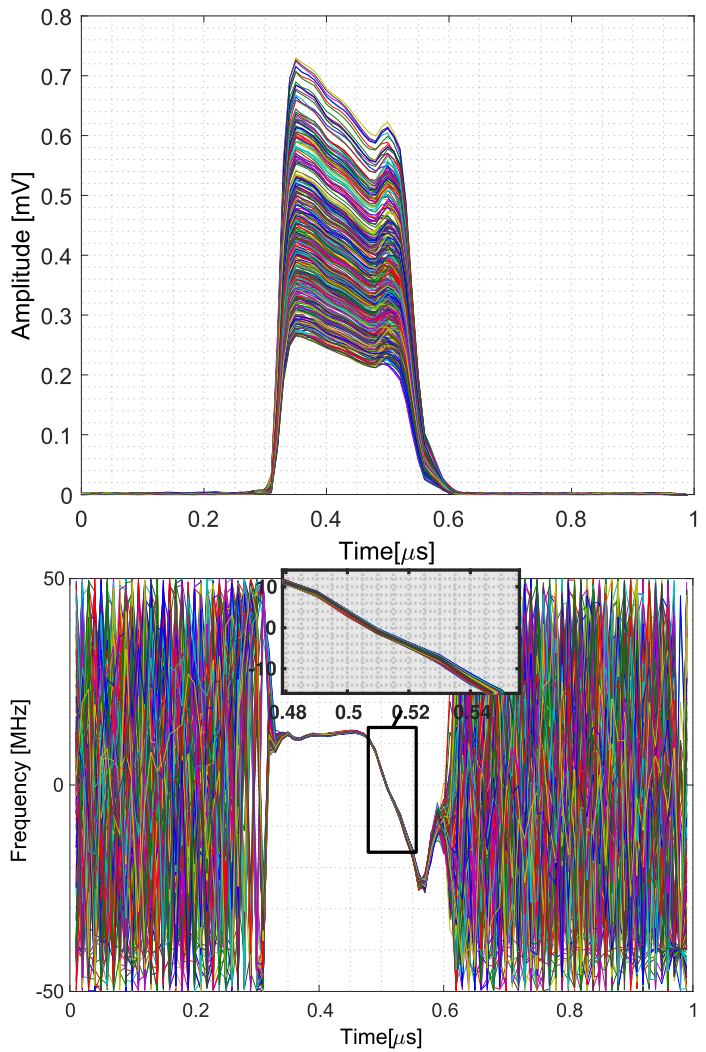

Fig. 2. Envelopes (above) and instantaneous frequencies (below) of the aligned pulses, whose original versions are shown in Fig. 1.

where $\mathrm{SNR}_{\min }$ and $\mathrm{SNR}_{\max }$ denote the SNR of the pulses with the highest and lowest SNR respectively. Hence, assuming that the amplitudes of all the pulses are about the same, the SNR increase would be $10 \log _{10}(P) \mathrm{dB}$.

Assuming that $r, 1 \leq r \leq P$, is the index of the reference pulse, and that the first $K$ samples are used in alignment, define:

$$
\begin{aligned}
& \mathbf{y}_{k, K}=\left[g_{r}\left(t_{k}\right), g_{r}\left(t_{k+1}\right), . ., g_{r}\left(t_{k+K-1}\right)\right]^{T}, \\
& \mathbf{x}_{k, K}=\left[g_{p}\left(t_{k}\right), g_{p}\left(t_{k+1}\right), . ., g_{p}\left(t_{k+K-1}\right)\right]^{T},
\end{aligned}
$$

where $\mathbf{y}_{k, K}$ and $\mathbf{x}_{k, K}$ denote the vector of $K$ complex samples of the reference pulse $g_{r}\left(t_{n}\right)$ and the $p^{\text {th }}$ pulse $g_{p}\left(t_{n}\right)$, respectively. The relative time delay and complex scaling factor that compensates for the phase and amplitude difference between these two pulses can be obtained as:

$$
\left[\tau^{*}, c^{*}\right]=\underset{[\tau, c]}{\arg \min }\left\|\mathbf{y}_{k, K}-c \mathbf{x}_{k, K}(\tau)\right\|_{l_{2}}^{2},
$$

where $\mathbf{x}_{k, K}(\tau)=\left[g_{p}\left(t_{k}+\tau\right), g_{p}\left(t_{k+1}+\tau\right), . ., g_{p}\left(t_{k+K-1}+\tau\right)\right]^{T}$, and $\|\cdot\|_{l_{2}}$ denotes the $l_{2}$ norm operator. For a given $\tau$, the optimal value of $c$ can be found as:

$$
c(\tau)=\mathbf{x}_{k, K}(\tau)^{H} \mathbf{y}_{k, K} /\left\|\mathbf{x}_{k, K}(\tau)\right\|_{l_{2}}^{2} .
$$

Hence, the required two-dimensional optimization in (8) reduces to a 1-dimensional optimization problem:

$$
t^{*}=\underset{\tau}{\arg \min }\left\|\mathbf{y}_{k, K}-c(\tau) \mathbf{x}_{k, K}(\tau)\right\|_{l_{2}}^{2} .
$$

Since the cost function in (10) is non-convex, its cost surface might have multiple local minima. In terms of the sampling interval, $T_{s}=1 / F_{S}$, where $F_{s}$ is the sampling frequency, $\tau$ can be written as:

$$
\tau=\zeta T_{s}+\gamma T_{s},
$$

where $0<\gamma<1$ and $\zeta=\left\lfloor\tau / T_{s}\right\rfloor$, where $\lfloor$.」 denotes the flooring operator. In this expression, $\zeta T_{s}$ is a coarse estimate of $\tau$ whose resolution is limited by the sampling interval of the receiver, and $\gamma T_{s}$ is a fine resolution correction.

For the estimation of these two parameters, we propose a two-stage algorithm that avoids getting trapped in a local minimum of the cost function given in (10). In the first stage, a coarse search is utilized for estimating $\zeta$. In the second stage a fine search is utilized for estimating $\gamma$. In the subsections below, we detail these two stages.

\section{A. Coarse Search: Estimation of $\zeta$}

Assuming that fine resolution correction term is $\gamma=0$ in (11), the cost function given in (10) can be written in terms of the coarse estimate $\zeta$ as:

$$
f_{\zeta}(\zeta) \equiv-2 \operatorname{Re}\left\{c(\zeta) \mathbf{y}_{k, K}^{H} \mathbf{x}_{k, K}(\zeta)\right\}+|c(\zeta)|^{2}\left\|\mathbf{x}_{k, K}(\zeta)\right\|^{2}
$$

where $c(\zeta)$ is as in (9). Since $\zeta \in \mathcal{Z}, \mathbf{x}_{k, K}(\zeta)$ becomes:

$$
\begin{aligned}
\mathbf{x}_{k, K}(\zeta) & =\mathbf{x}_{k+\zeta, K} \\
& =\left[g_{p}\left(t_{k+\zeta}\right), g_{p}\left(t_{k+1+\zeta}\right), . ., g_{p}\left(t_{k+K-1+\zeta}\right)\right]^{T} .
\end{aligned}
$$

Since all the pulses are coarsely aligned according to their TOA values by the receiver, the cost function in (12) can be calculated over a limited interval $-\kappa \leq \zeta \leq \kappa$, and the minimum of the cost function $\bar{\zeta}$ can be found. Note that the coarse alignment term $\zeta$ given in (11) is smaller than the actual delay value $\tau$, and that the fine resolution correction term $\gamma$ is always positive. As a result, the coarse estimate value is computed as:

$$
\hat{\zeta}=\lambda(\bar{\zeta})= \begin{cases}\bar{\zeta}-1 & \text { if } f_{\zeta}(\bar{\zeta}-1) \leq f_{\zeta}(\bar{\zeta}+1), \\ \bar{\zeta} & \text { if } f_{\zeta}(\bar{\zeta}-1)>f_{\zeta}(\bar{\zeta}+1)\end{cases}
$$

\section{B. Fine Search: Estimation of $\gamma$}

In the coarse estimation stage of $\zeta$, the time offset whose resolution is limited by the sampling interval is estimated. In the fine search stage, the coarsely-estimated time delay is updated in order to precisely align pulses beyond the sampling frequency, by minimizing the following simplified cost function:

$$
f_{\gamma}(\gamma)=-2 \operatorname{Re}\left\{c(\gamma) \mathbf{y}_{k, K}^{H} \mathbf{x}_{\hat{k}, K}(\gamma)\right\}+|c(\gamma)|^{2}\left\|\mathbf{x}_{\hat{k}, K}(\gamma)\right\|^{2} .
$$

Here $c(\gamma)$ is as in (9), $\hat{k}=k+\hat{\zeta}$ and $\mathbf{x}_{\hat{k}, K}(\gamma)$ is defined as:

$$
\mathbf{x}_{\hat{k}, K}(\gamma)=\left[g_{p}\left(t_{\hat{k}}+\gamma\right), g_{p}\left(t_{\hat{k}+1}+\gamma\right), \cdot \cdot, g_{p}\left(t_{\hat{k}+K-1}+\gamma\right)\right]^{T} .
$$

To obtain $\mathbf{x}_{\hat{k}, K}(\gamma)$, a cubic spline interpolator on the available samples can be used [21]. To simplify the notation, let us 


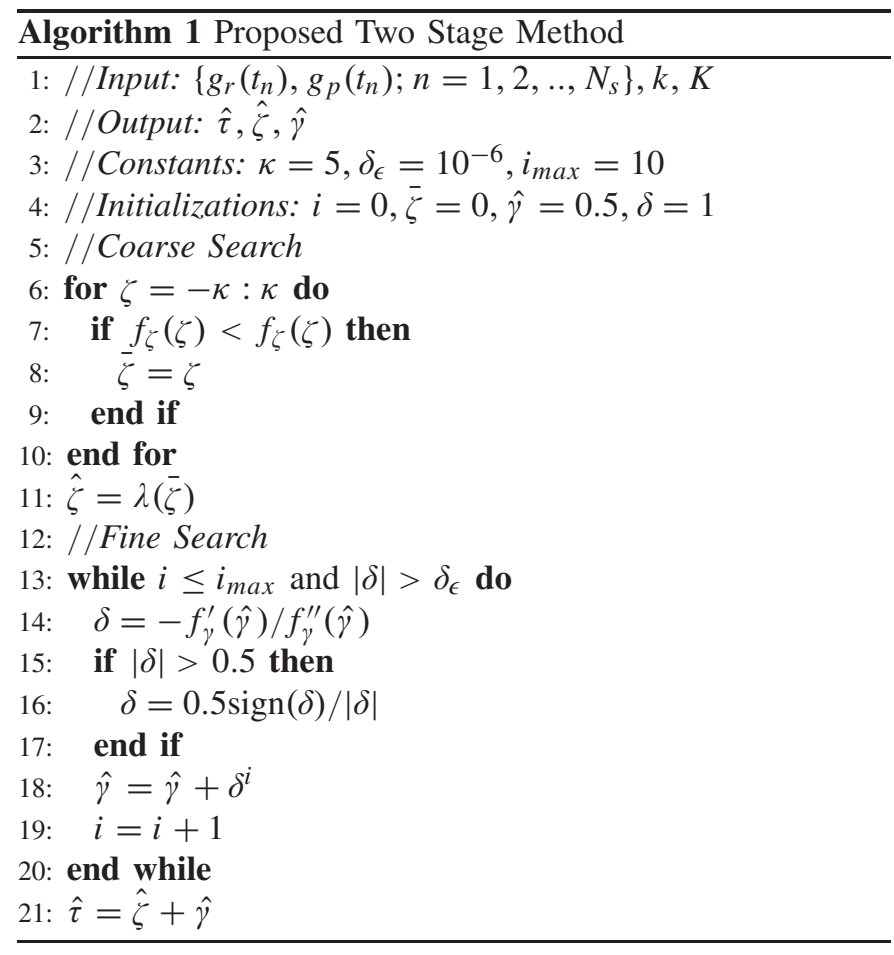

define $\hat{\mathbf{x}} \triangleq\left[g_{p}\left(t_{\hat{k}}\right), g_{p}\left(t_{\hat{k}+1}\right), . ., g_{p}\left(t_{\hat{k}+K}\right)\right]^{T}$, whose entries are modeled as the discrete samples of the following continuous function:

$$
s_{\hat{\mathbf{x}}, n}(\gamma)=a_{n}+b_{n} \gamma+c_{n} \gamma^{2}+d_{n} \gamma^{3}, 1 \leq n \leq K,
$$

where $\gamma \in[0,1]$. The spline parameters $a_{n}, b_{n}, c_{n}, d_{n}$ are found by solving for $\mathbf{h}$ in the following linear system [21]:

$$
\mathbf{A h}=\mathbf{v},
$$

where $\mathbf{A} \in \mathcal{R}^{(K+1) \times(K+1)}$ and $\mathbf{v} \in \mathcal{C}^{K+1}$ are defined as:

$$
\mathbf{A}=\left[\begin{array}{cccccc}
2 & 1 & 0 & 0 & . \cdot & 0 \\
1 & 4 & 1 & 0 & . . & 0 \\
0 & 1 & 4 & 1 & . \cdot & 0 \\
. & . & . & . & . & \\
0 & . . & 0 & 1 & 4 & 1 \\
0 & . \cdot & 0 & 0 & 2 & 1
\end{array}\right], \mathbf{v}=\left[\begin{array}{c}
3\left(\hat{\mathbf{x}}_{2}-\hat{\mathbf{x}}_{1}\right) \\
3\left(\hat{\mathbf{x}}_{3}-\hat{\mathbf{x}}_{1}\right) \\
3\left(\hat{\mathbf{x}}_{4}-\hat{\mathbf{x}}_{2}\right) \\
\vdots \\
3\left(\hat{\mathbf{x}}_{K+1}-\hat{\mathbf{x}}_{K-1}\right) \\
3\left(\hat{\mathbf{x}}_{K+1}-\hat{\mathbf{x}}_{K}\right)
\end{array}\right]
$$

Here $\hat{\mathbf{x}}_{i}$ represents the $i^{\text {th }}$ entry of $\hat{\mathbf{x}}$. Since the coefficient matrix $\mathbf{A}$ is tridiagonal, the linear system in (18) can be solved for $\mathbf{h}$ efficiently in $\mathcal{O}(K+1)$ complexity by using the Thomas Algorithm [22]. Then the spline parameters for $1 \leq n \leq K$ are obtained as [21]:

$$
\begin{aligned}
a_{n} & =\hat{\mathbf{x}}_{n}, \\
b_{n} & =\mathbf{h}_{n}, \\
c_{n} & =3\left(\hat{\mathbf{x}}_{n+1}-\hat{\mathbf{x}}_{n}\right)-2 \mathbf{h}_{n}-\mathbf{h}_{n+1}, \\
d_{n} & =2\left(\hat{\mathbf{x}}_{n}-\hat{\mathbf{x}}_{n+1}\right)+\mathbf{h}_{n}+\mathbf{h}_{n+1} .
\end{aligned}
$$

Then, by using the following $\mathbf{B}_{\hat{k}, K} \in \mathcal{C}^{K \times 4}$ matrix

$$
\mathbf{B}_{\hat{k}, K}=\left[\begin{array}{cccc}
a_{1} & b_{1} & c_{1} & d_{1} \\
a_{2} & b_{2} & c_{2} & d_{2} \\
\cdot & & & \\
a_{K} & b_{K} & c_{K} & d_{K}
\end{array}\right],
$$

the vector $\mathbf{x}_{\hat{k}, K}(\gamma)$ can be expressed as a third order piece-wise continuous polynomial:

$$
\mathbf{x}_{\hat{k}, K}(\gamma)=\mathbf{B}_{\hat{k}, K} \boldsymbol{\gamma}
$$

where $\gamma=\left[1, \gamma, \gamma^{2}, \gamma^{3}\right]^{T}$. By substituting (22) into (15), the following cost function is obtained:

$$
f_{\gamma}(\gamma)=-2 \operatorname{Re}\left\{c(\gamma) \mathbf{y}_{k, K}^{H} \mathbf{B}_{\hat{k}, K} \boldsymbol{\gamma}\right\}+|c(\gamma)|^{2}\left\|\mathbf{B}_{\hat{k}, K} \boldsymbol{\gamma}\right\|^{2} .
$$

To find the local minimum of $f_{\gamma}(\gamma)$, the Newton-Raphson algorithm can be used [22]. In the required iterations, the first and second order derivatives of $f_{\gamma}(\gamma)$ are required. To compute these derivatives, let us rewrite $f_{\gamma}(\gamma)$ as

$$
f_{\gamma}(\gamma)=-2 \operatorname{Re}\{c(\gamma) p(\gamma)\}+g(\gamma) q(\gamma),
$$

where $p(\gamma)=\mathbf{y}_{k, K}^{H} \mathbf{B}_{\hat{k}, K} \boldsymbol{\gamma}, q(\gamma)=\boldsymbol{\gamma}^{T} \mathbf{B}_{\hat{k}, K}^{H} \mathbf{B}_{\hat{k}, K} \boldsymbol{\gamma}$ and $g(\gamma)=|c(\gamma)|^{2}$. Using these definitions, $f_{\gamma}^{\prime}(\gamma)$ and $f_{\gamma}^{\prime \prime}(\gamma)$ are computed as:

$$
\begin{aligned}
f_{\gamma}^{\prime}(\gamma)= & -2 \operatorname{Re}\left\{c^{\prime}(\gamma) p(\gamma)+c(\gamma) p^{\prime}(\gamma)\right\} \\
& +g^{\prime}(\gamma) q(\gamma)+g(\gamma) q^{\prime}(\gamma), \\
f_{\gamma}^{\prime \prime}(\gamma)= & -2 \operatorname{Re}\left\{c^{\prime \prime}(\gamma) p(\gamma)+2 c^{\prime}(\gamma) p^{\prime}(\gamma)+c(\gamma) p^{\prime \prime}(\gamma)\right\} \\
& +g^{\prime \prime}(\gamma) q(\gamma)+2 g^{\prime}(\gamma) q^{\prime}(\gamma)+g(\gamma) q^{\prime \prime}(\gamma),
\end{aligned}
$$

where the following closed form expressions for the derivatives are used in the iterations:

$$
\begin{aligned}
p^{\prime}(\gamma)= & \mathbf{y}_{k, K}^{H} \mathbf{B}_{\hat{k}, K} \mathbf{D} \boldsymbol{\gamma}, \\
p^{\prime \prime}(\gamma)= & \mathbf{y}_{k, K}^{H} \mathbf{B}_{\hat{k}, K} \mathbf{D}^{2} \boldsymbol{\gamma}, \\
q^{\prime}(\gamma)= & \boldsymbol{\gamma}^{T}\left(\mathbf{D}^{T} \mathbf{B}_{\hat{k}, K}^{H} \mathbf{B}_{\hat{k}, K}+\mathbf{B}_{\hat{k}, K}^{H} \mathbf{B}_{\hat{k}, K} \mathbf{D}\right) \boldsymbol{\gamma}, \\
q^{\prime \prime}(\gamma)= & \boldsymbol{\gamma}^{T}\left(\left(\mathbf{D}^{T}\right)^{2} \mathbf{B}_{\hat{k}, K}^{H} \mathbf{B}_{\hat{k}, K}+2 \mathbf{D}^{T} \mathbf{B}_{\hat{k}, K}^{H} \mathbf{B}_{\hat{k}, K} \mathbf{D}\right. \\
& \left.+\mathbf{B}_{\hat{k}, K}^{H} \mathbf{B}_{\hat{k}, K} \mathbf{D}^{2}\right) \boldsymbol{\gamma},
\end{aligned}
$$

where $\mathbf{D}$ is the matrix that obtains the derivative of vector $\gamma=\left[1, \gamma, \gamma^{2}, \gamma^{3}\right]^{T}$. Using $p(\gamma)$ and $q(\gamma), c(\gamma)$ can be written as $c(\gamma)=p^{*}(\gamma) / q(\gamma)$. Then, the first and second order derivatives of $c(\gamma)$ are obtained as:

$$
\begin{aligned}
c^{\prime}(\gamma)= & \frac{p^{\prime *}(\gamma)}{q(\gamma)}-\frac{q^{\prime}(\gamma) p^{*}(\gamma)}{q(\gamma)^{2}}, \\
c^{\prime \prime}(\gamma)= & \frac{p^{\prime \prime *}(\gamma)}{q(\gamma)}-\frac{q^{\prime}(\gamma) p^{\prime *}(\gamma)}{q(\gamma)^{2}} \\
& -\frac{q^{\prime \prime}(\gamma) p^{*}(\gamma)+p^{\prime *}(\gamma) q^{\prime}(\gamma)}{q(\gamma)^{2}} \\
& +2 \frac{\left(q^{\prime}(\gamma)\right)^{2} p^{*}(\gamma)}{q(\gamma)^{3}} .
\end{aligned}
$$

Finally, the derivatives of $g(\gamma)$ can be written in terms of $c(\gamma)$ and $c^{\prime}(\gamma)$ as:

$$
\begin{aligned}
g^{\prime}(\gamma) & =2 \operatorname{Re}\left\{c^{\prime}(\gamma) c^{*}(\gamma)\right\} \\
g^{\prime \prime}(\gamma) & =2 \operatorname{Re}\left\{c^{\prime \prime}(\gamma) c^{*}(\gamma)\right\}+\left|c^{\prime}(\gamma)\right|^{2} .
\end{aligned}
$$


The above expressions for the derivatives lead to accurate convergence in about 20 iterations.

Once $\hat{\gamma}$ and $\hat{\zeta}$ are obtained, $\tau$ is estimated as:

$$
\hat{\tau}=(\hat{\gamma}+\hat{\zeta}) / F_{S},
$$

where $\hat{\zeta}$ and $\hat{\gamma}$ are the minimizers of (12) and (23), respectively. The overall algorithm is summarized in Alg-1. Once the relative delays of all pulses with respect to the reference pulse are estimated, all the pulses are coherently integrated using (4). In the integration process, estimated delay values, which are at a higher resolution than the sampling interval, can be handled either by using the continuous function model given in (17) or by using fractional-delay filters [21]. In the next section, we present results obtained on both synthetic and real data sets.

\section{Experimental Results on Pulse Alignment}

To investigate the performance of the proposed delay estimation method, we performed experiments on the following synthetically generated radar pulses:

$$
g(t)=A a(t) e^{j \phi(t)},
$$

where $A$ is the amplitude, $\phi(t)$ is the instantaneous phase, and $a(t)$ is the envelope of the pulse defined as:

$$
a(t)= \begin{cases}e^{-t^{2} / \sigma_{T R}^{2}} & \text { if } t \leq 0, \\ 1 & \text { if } 0<t \leq T_{P W}, \\ e^{\left(-t-T_{P W}\right)^{2} / \sigma_{T F}^{2}} & \text { if } t>T_{P W},\end{cases}
$$

where the pulse width $T_{P W}$ is chosen as $1 \mu s$ and both the pulse rise time $\sigma_{T R}$ and pulse fall time $\sigma_{T F}$ are chosen as $0.01 \mu s$. Each pulse is modulated by using the following frequency modulation:

$$
f_{g}(t)=0.5 B_{g} \cos \left(2 \pi t / T_{g}\right),
$$

where $B_{g} \in\{1,2,4,8,16,32\} \mathrm{MHz}$ and $T_{g}=1 \mu \mathrm{s}$ are the FM deviation (bandwidth) and the FM rate of the modulation. The corresponding instantaneous phase of the pulse is:

$$
\phi(t)=\int_{-\infty}^{t} f_{g}(\hat{t}) d \hat{t}+\phi_{0},
$$

where $\phi_{0}$ denotes the frequency offset of the pulse. The sampling frequency of the waveform is set to $F_{s}=100$ MHz. Finally, circularly symmetric white Gaussian noise with appropriately chosen standard deviation $\sigma_{z}$ is added to set the SNR to any desired value, given by SNR $=20 \log 10\left(A / \sigma_{z}\right)$ $\mathrm{dB}$. At each SNR and modulation bandwidth $B_{g}, 10000$ Monte-Carlo runs were performed. In each run, a delayed pulse $g(t-\tau / F s)$ is generated where $\tau$ is chosen from a uniform random distribution in the interval $[-5,5]$. Its phase offset $\phi_{0}$ is chosen from a uniform random distribution in the interval $[-\pi, \pi)$. The standard deviation of the estimation error as a function of SNR and $B_{g}$ is shown in Fig. 3. As observed, the proposed method performs better with increasing SNR and achieves a minimum estimation error of $10^{-5.4}$ samples at $105 \mathrm{~dB}$. Beyond this point, there is no performance improvement, since the modeling error in representing the discrete measurements by cubic splines

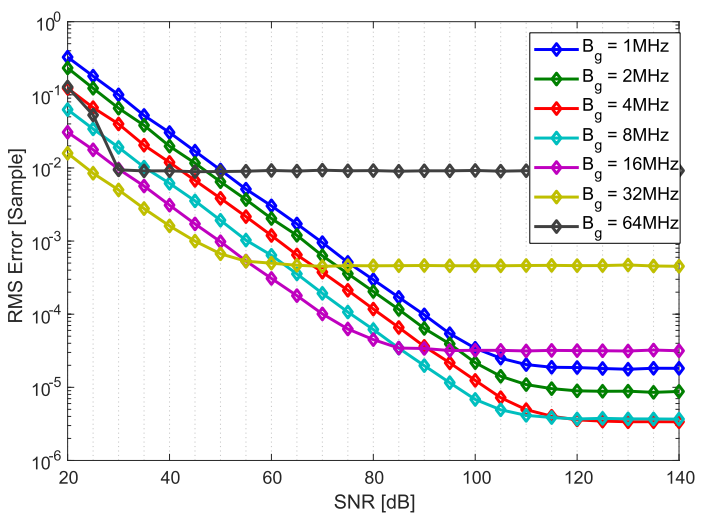

Fig. 3. Standard deviation of the delay estimation error as a function of SNR, for different values of $B_{g}$.

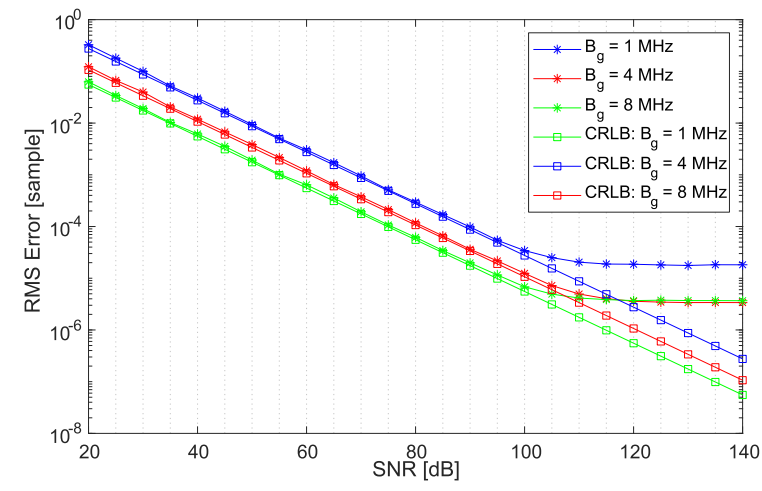

Fig. 4. Standard deviation of the delay estimation error and CRLB as a function of SNR and bandwidth $B_{g}$.

becomes dominant. Similar behavior can also be seen in Fig. 4, where the Cramer-Rao lower bound (CRLB) for estimating the time delay between two measurements given in [23] is also shown. Another important observation is that increasing the $\mathrm{BW}$ of the pulse for a fixed FM rate improves the estimation performance, which is in agreement with the CRLB. However, beyond a value of $16 \mathrm{MHz}$, performance drops significantly. This observation can be explained as follows. As the BW increases, the non-regularity in the pulse increases, hence the algorithm performs better. However, at very high BW, the instantaneous frequency of the pulse gets closer to the Nyquist limit $\left(F_{S} / 2\right)$, and the cubic splines become insufficient in representing the available samples.

We also performed experiments on a real data set. The pulses shown in Fig. 1 are aligned by using the proposed technique and the results are shown in Fig. 2. As compared to Fig. 1, the instantaneous frequency curves of the aligned pulses are significantly closer to each other for every time instant. This effect is more evident when there exist some frequency modulations in the pulse.

To demonstrate the importance of pulse integration for precise SEI analysis, a third experiment is conducted, where the response of a T-module is measured, which is the basic transmitting element in phased-array radars. We applied a $1 \mu \mathrm{s}$ pulse at a frequency of $8 \mathrm{GHz}$ to the input of a T-module 

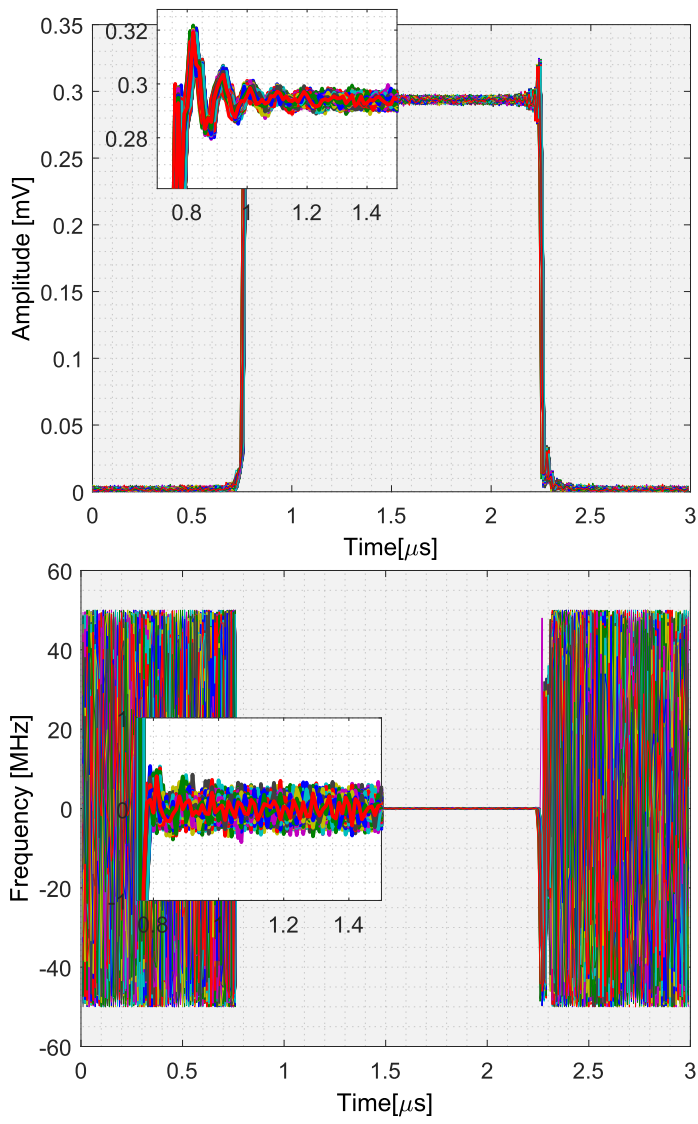

Fig. 5. Envelopes (above) and instantaneous frequencies (below) of the pulses emitted by a T-module.

and collected 500 pulses from its output. The envelopes and instantaneous frequencies of the pulses are shown in Fig. 5. In each plot, the close-up inserts are also provided. First, available pulses are aligned by using the proposed method and then integrated using (4). The resulting integrated pulse is shown in Fig. 6. In the close-up insert region of the instantaneous frequency graph, there appears to be some unintentional frequency modulation with a bandwidth of a few $\mathrm{kHz}$. Note that this modulation is not visible prior to pulse integration.

\section{RADAR FINGERPRINT EXTRACTION}

In this section we will provide details for radar fingerprint extraction. To illustrate the result of each stage we will use real measurements of four solid state radar power amplifiers that are manufactured on the same production line. During the experiments, 500 pulses are obtained from each power amplifier, and input signals driving the amplifiers are set so that the outputs of the amplifiers are saturated. In Fig. 7, measurements obtained for two different amplifiers that will be referred to as Amp-1 and Amp-2 are shown. Comparing the experimental data we have obtained, unintentional modulations on real data can be observed at the on-set of each pulse. As a result, we used the time window with duration of $1 \mu \mathrm{s}$ around the point where the pulse detection first occurs. Considering this fact, we take a closer look at the time window shown in Fig.7 after subtracting an ideal square wave that fits the
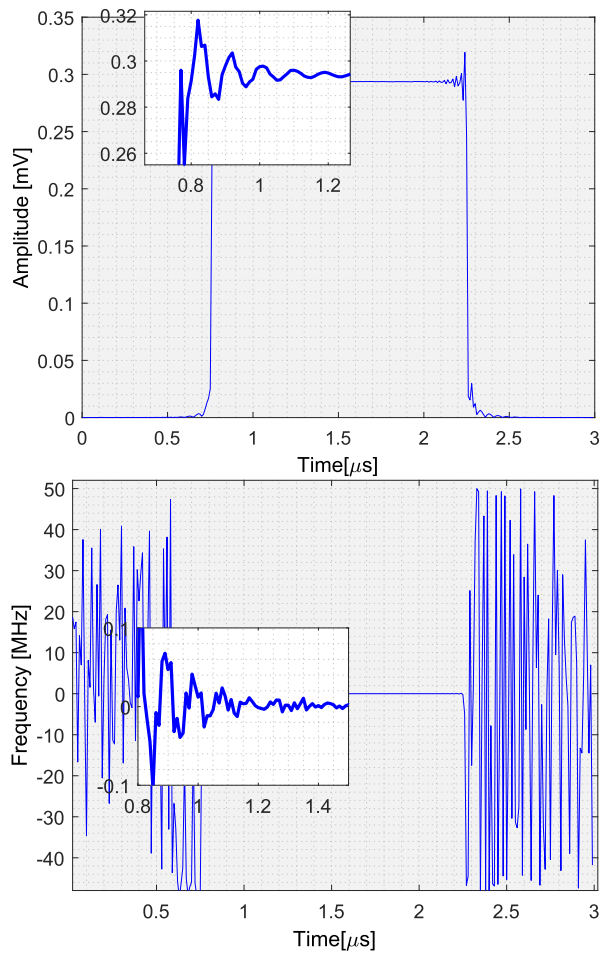

Fig. 6. Envelope (above) and instantaneous frequency (below) of the pulse obtained by integrating all the pulses shown in Fig. 5.

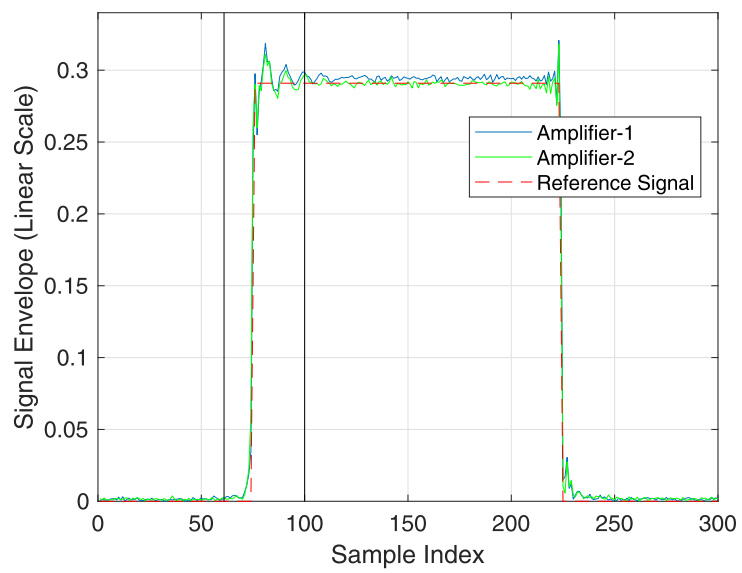

Fig. 7. Blue: Amplifier-1 amplitude; Green: Amplifier-2 amplitude; Red: Reference ideal square wave signal that will be subtracted from the original signals; Black: Time window for UMOP.

measurement data. Residual signals are shown in Fig. 8 and Fig. 9, respectively.

In order to observe localized frequency characteristics of the residual signal $r[n]$, the Short Time Fourier Transform (STFT) can be used [24]:

$$
R(m, w)=\sum_{n=-\infty}^{\infty} r[n] w[m-n] e^{-j w n}
$$

where $R(m, w)$ is the STFT of $r[n]$ at time $m$ and $w[n]$ is the window function. By using the Fast Fourier Transform (FFT), the discrete STFT can be computed at $w=\frac{2 \pi}{N} k, 0 \leq k \leq N-1$. 

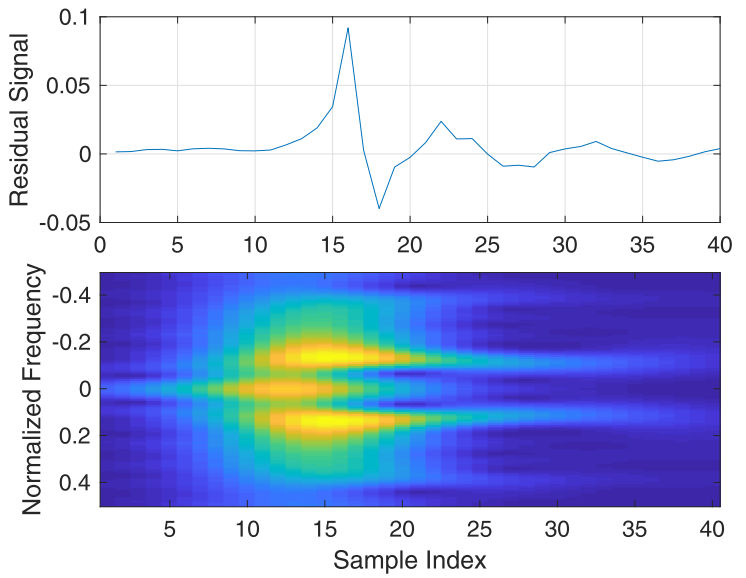

Fig. 8. Residual Amp-1 signal after subtracting the square wave (top) and its STFT (bottom).
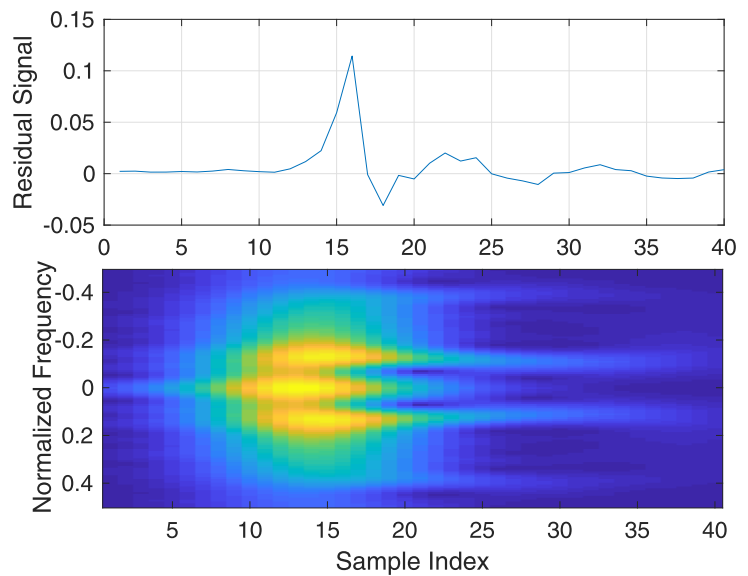

Fig. 9. Residual Amp-2 signal after subtracting the square wave (top) and its STFT (bottom).

In Fig. 8 and Fig. 9, the discrete STFTs of the residual signals are shown for a Gaussian window function with standard deviation of $\sigma=5$. Although these two signals are very similar in the time domain, more prominent differences can be observed in their STFTs. Both signals have two significant components with different time-frequency supports. For example, in Fig. 8, the frequency support of each component is more compact compared to the frequency support of the corresponding components in Fig. 9. Also, the time differences between these components are not the same for Amp-1 and Amp-2. Therefore, features generated using these components with compact frequency and time support have the potential to serve as the fingerprint. This requires robust estimation of these components from the original signal. For this purpose, the Variational Mode Decomposition (VMD) which as introduced in [20] can be used. In VMD, all modes are estimated concurrently by using the Alternating Direction Method of Multipliers (ADMM) optimization technique [25]. In the remainder of this section, we provide a brief review of the VMD based on [20], and detail its use in fingerprint extraction.

The goal of VMD is to decompose a real valued input signal $x(t)$ into a number of modes, $u_{k}(t)$, that have a compact
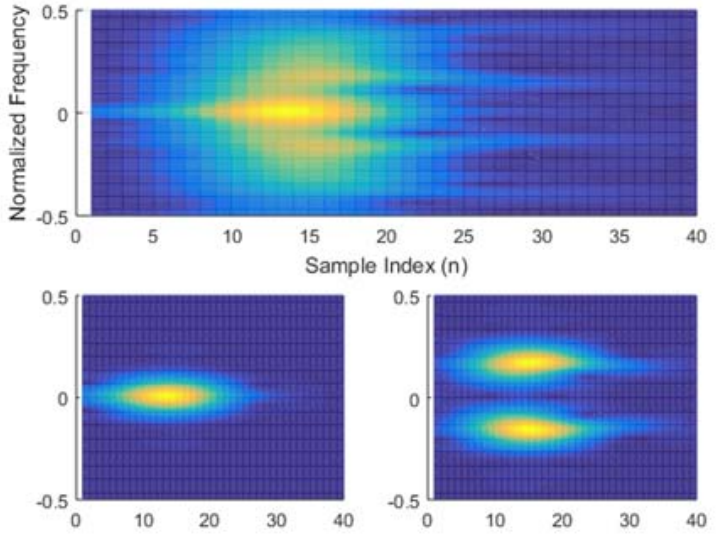

Fig. 10. Amp-2 signal after decomposing into mods using VMD.

support around a center frequency $w_{k}$ :

$$
u_{k}(t)=A_{k}(t) \cos \left(\phi_{k}(t)\right)
$$

where the modes $u_{k}(t)$ are identified as the solution to the following optimization problem:

$$
\begin{aligned}
\min _{\left\{u_{k}(t)\right\},\left\{w_{k}\right\}} & \left(\sum_{k}\left\|\partial_{t}\left[\left(\delta(t)+\frac{j}{\pi t}\right) * u_{k}(t)\right] e^{-j w_{k} t}\right\|_{2}^{2}\right) \\
\text { s.t. } & \sum_{k} u_{k}(t)=x(t) .
\end{aligned}
$$

Here, $\left\{u_{k}(t)\right\}:=\left\{u_{1}(t), u_{2}(t), \ldots, u_{k}(t)\right\}$ and $\left\{w_{k}\right\}:=$ $\left\{w_{1}, w_{2}, \ldots, w_{k}\right\}$ denotes the center frequency of each mode.

In order to address the constraint of the optimization problem given in (42), both the quadratic penalty term and the Lagrange multipliers are used by the VMD. The quadratic penalty term is used to boost the fidelity of the decomposition in the presence of noise while the Lagrange multipliers forces the constraint to be met. The augmented Lagrangian can be written as:

$$
\begin{gathered}
\mathcal{L}\left(\left\{u_{k}\right\},\left\{w_{k}\right\}, \lambda\right):=\alpha \sum_{k}\left\|\partial_{t}\left[\left(\delta(t)+\frac{j}{\pi t}\right) * u_{k}(t)\right] e^{-j w_{k} t}\right\|_{2}^{2} \\
+\left\|f(t)-\sum_{k} u_{k}(t)\right\|_{2}^{2}+\left\langle\lambda(t), f(t)-\sum_{k} u_{k}(t)\right\rangle \cdot
\end{gathered}
$$

The solution of the optimization problem given in (43) can be found as a saddle point of the augmented Lagrangian $\mathcal{L}$ in a sequence of iterative sub-optimizations by using the ADMM technique. Steps of the algorithm is given in Algorithm 2. Detailed derivations of the VMD technique can be found in [20].

There are two important points that should be emphasized for VMD. In (44), similar to the expectation maximization approach, the $k^{t h}$ mod signal is updated by Wiener filtering the residual signal that is obtained after removing the latest updated versions of the remaining mod signals. This filtering operation denoise the signal, and help us improve the SNR. 
TABLE I

Features Calculated for Each Mod Signal

\begin{tabular}{|l|l|l|}
\hline Feature & Time Domain Features & Frequency Domain Features \\
\hline Mean & $\mu_{k}^{t}=\frac{\int_{-\infty}^{\infty} t\left|u_{k}(t)\right|^{2} d t}{\int_{-\infty}^{\infty}\left|u_{k}(t)\right|^{2} d t}$ & $\mu_{k}^{w}=\frac{\int_{0}^{\infty} w\left|\hat{u}_{k}(w)\right|^{2} d w}{\int_{0}^{\infty}\left|\hat{u}_{k}(w)\right|^{2} d w}$ \\
\hline Standard Deviation & $\sigma_{k}^{t}=\left(\frac{\int_{-\infty}^{\infty}\left(t-\mu_{k}^{t}\right)^{2}\left|u_{k}(t)\right|^{2} d t}{\int_{-\infty}^{\infty}\left|u_{k}(t)\right|^{2} d t}\right)^{\frac{1}{2}}$ & $\sigma_{k}^{w}=\left(\frac{\int_{0}^{\infty}\left(w-\mu_{k}^{w}\right)^{2}\left|\hat{u}_{k}(w)\right|^{2} d w}{\int_{0}^{\infty}\left|\hat{u}_{k}(w)\right|^{2} d w}\right)^{\frac{1}{2}}$ \\
\hline Differential Entropy & $h_{k}^{t}=\frac{\int_{-\infty}^{\infty}\left|u_{k}(t)\right|^{2} \log \left(1 /\left|u_{k}(t)\right|^{2}\right) d t}{\int_{-\infty}^{\infty}\left|u_{k}(t)\right|^{2} d w}$ & $h_{k}^{w}=\frac{\int_{-\infty}^{\infty}\left|\hat{u}_{k}(w)\right|^{2} \log \left(1 /\left|\hat{u}_{k}(w)\right|^{2}\right) d w}{\int_{-\infty}^{\infty}\left|\hat{u}_{k}(w)\right|^{2} d w}$ \\
\hline Kurtosis & $\kappa_{k}^{t}=\frac{\int_{-\infty}^{\infty}\left(t-\mu_{k}^{t}\right)^{4} /\left(\sigma_{k}^{t}\right)^{4}\left|u_{k}(t)^{2}\right| d t}{\int_{-\infty}^{\infty}\left|u_{k}(t)\right|^{2} d t}$ & $\kappa_{k}^{w}=\frac{\int_{-\infty}^{\infty}\left(w-\mu_{k}^{w}\right)^{4} /\left(\sigma_{k}^{w}\right)^{4}\left|\hat{u}_{k}(w)^{2}\right| d w}{\int_{-\infty}^{\infty}\left|\hat{u}_{k}(w)\right|^{2} d w}$ \\
\hline Skewness & $s_{k}^{t}=\frac{\int_{-\infty}^{\infty}\left(t-\mu_{k}^{t}\right)^{3} /\left(\sigma_{k}^{t}\right)^{3}\left|u_{k}(t)^{2}\right| d t}{\int_{-\infty}^{\infty}\left|u_{k}(t)\right|^{2} d t}$ & $s_{k}^{w}=\frac{\int_{-\infty}^{\infty}\left(w-\mu_{k}^{w}\right)^{3} /\left(\sigma_{k}^{w}\right)^{3}\left|\hat{u}_{k}(w)^{2}\right| d w}{\int_{-\infty}^{\infty}\left|\hat{u}_{k}(w)\right|^{2} d w}$ \\
\hline
\end{tabular}

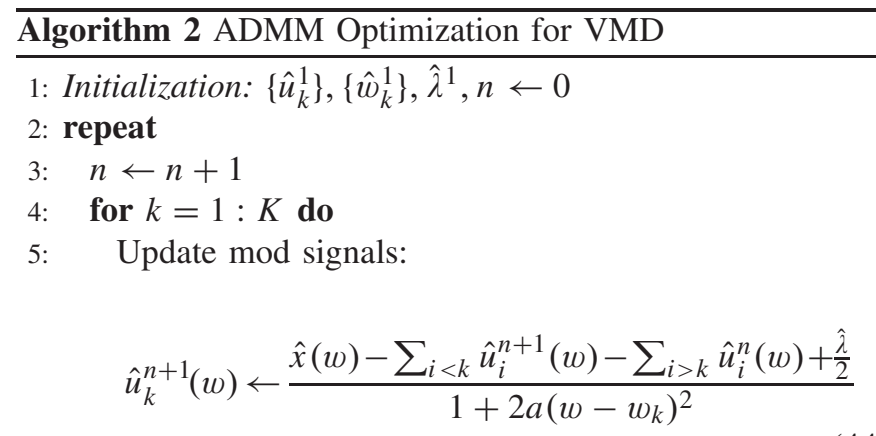

6: $\quad$ Update mod frequencies:

$$
w_{k}^{n+1} \leftarrow \frac{\int_{0}^{\infty} w\left|\hat{u}_{k}^{n+1}(w)\right|^{2} d w}{\int_{0}^{\infty}\left|\hat{u}_{k}^{n+1}(w)\right|^{2} d w}
$$

7: $\quad$ end for

8: Dual Ascent for all $w \geq 0$ :

$$
\hat{\lambda}^{n+1}(w) \leftarrow \hat{\lambda}^{n}(w)+\tau\left(\hat{x}(w)-\sum_{k} \hat{u}_{k}^{n+1}(w)\right)
$$

9: until convergence $\sum_{k}\left\|\hat{u}_{k}^{n+1}-\hat{u}_{k}^{n}\right\|_{2}^{2} /\left\|\hat{u}_{k}^{n}\right\|_{2}^{2}<\epsilon$

Secondly, in (45), $w_{k}$ is chosen as the center of gravity of the power spectrum of the $k^{\text {th }}$ mode.

The VMD technique can be used for the decomposition of UMOP signals into mods with compact frequency supports prior to feature extraction. An example of decomposed signals following the VMD is shown in Fig. 10. In order to characterize these mod signals, distribution of their energy in both time and frequency domains can be used. For example, the center of gravity of the mod signal in the time domain can be used to characterize the position of the mod signal, whereas in the frequency domain, the same approach would yield the estimate of its center frequency. This idea can be extended to generate the features given in Table. I, which are obtained by normalizing the energy of the mod signals and considering them as probability density functions (PDF).
Note that, all features except the time center of the mods are time invariant, i.e., extracted features are not affected by the time shift of the signal in the acquisition window. This is actually a desired property that improves the robustness of the feature extraction method, as the position of the pulse rise time in the data acquisition window might change slightly between different measurements. By using the mean time of the first mod signal as a reference, this feature can also be transformed to a time invariant feature as:

$$
\mu_{k}^{t_{r e f}}=\mu_{k}^{t}-\mu_{0}^{t}
$$

where $\mu_{0}^{t}$ is the mean time of the first mod signal.

\section{Computational Complexity Analysis}

In this section, the computational complexity of the proposed method will be analysed. It will be assumed that $N$ pulses each having $K$ samples are available. In the coarse alignment step, $\mathcal{O}(K)$ operations are performed for evaluating (12), hence $\kappa \times \mathcal{O}(K)$ operations are required for finding the coarse delay estimate $\zeta$. In the fine alignment step, the main complexity arises from solving the linear system of equations given in (18) and the derivatives defined from (27) to (30). Since $\mathbf{A}$ in (18) is tri-diagonal, it can be solved in $\mathcal{O}(K+1)$ operations using the Thomas Algorithm [22]. The consecutive derivatives of $p(\gamma)$ and $q(\gamma)$ can also be evaluated with $\mathcal{O}(K)$ operations. Hence, for $N$ pulses, the total complexity of the alignment procedure requires $\mathcal{O}(K \times N)$ operations.

In the VMD stage, $\mathcal{O}(K)$ operations are required in (44) and (45) in Algorithm 2. If $M$ mods are extracted (in our case $M=2$ ) and $T$ iterations are utilized ( $T$ is typically around 50 in our experiments), the total number operations required in the VMD stage is $\mathcal{O}(K \times M \times T)$. Note that, since this stage is utilized for the integrated pulse, the complexity of this stage is independent of the number of pulses. In the feature extraction step, all the features are computed at $\mathcal{O}(K)$ operations.

Overall, the main computational burden of the proposed method arises from the pulse alignment procedure, which is linear in the number of samples $K$ and the number of pulses $N$. 


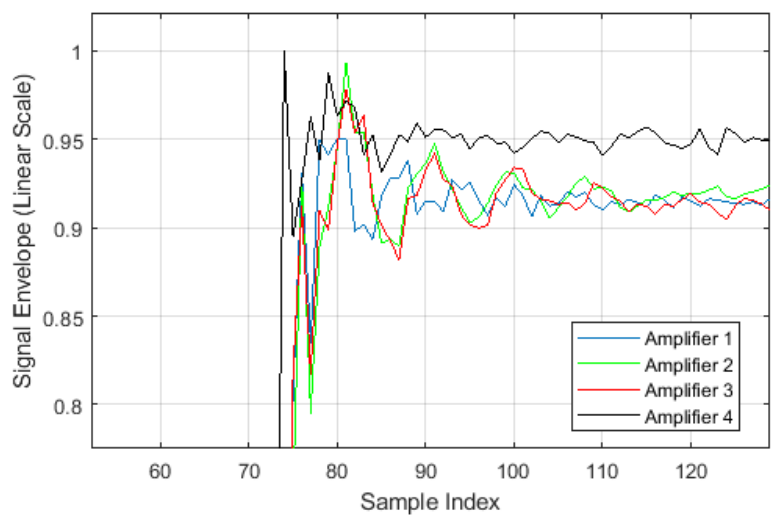

Fig. 11. Signal envelopes for 4 different amplifiers.

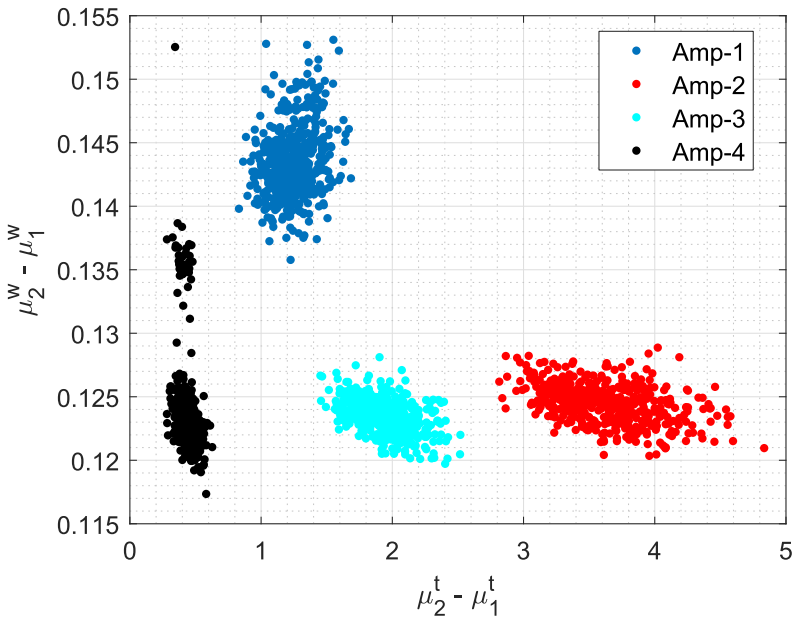

Fig. 12. Time and frequency center features.

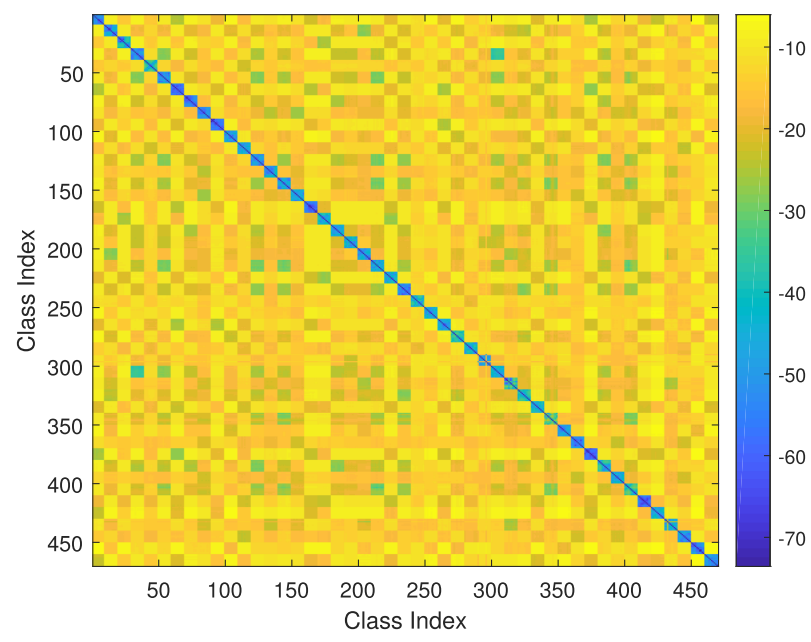

Fig. 13. Normalized Euclidean Distance of features.

\section{Results on REAl Field DATA}

To demonstrate the performance of the proposed SEI technique, three different data sets that consist of real measurements are used. During the experiments, the signals are decomposed into two components by VMD. The first set

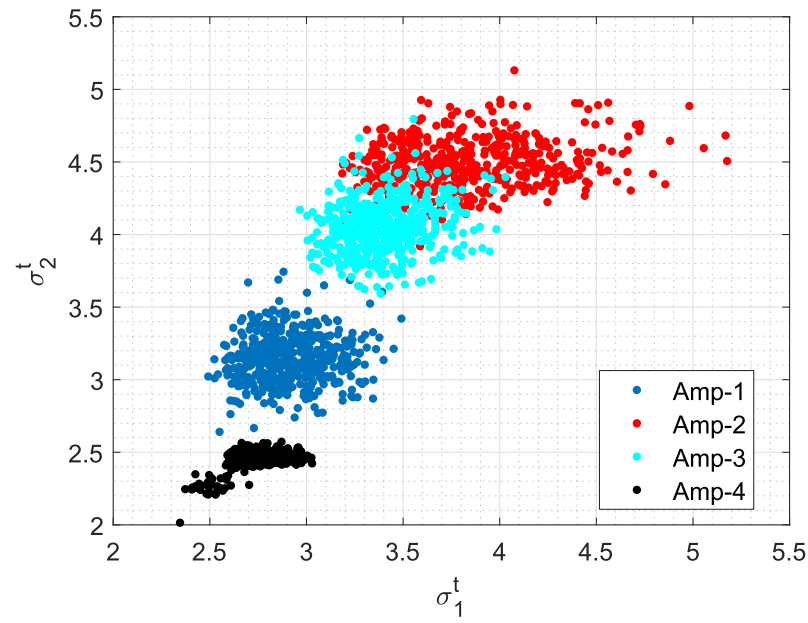

Fig. 14. Time standard deviation feature.

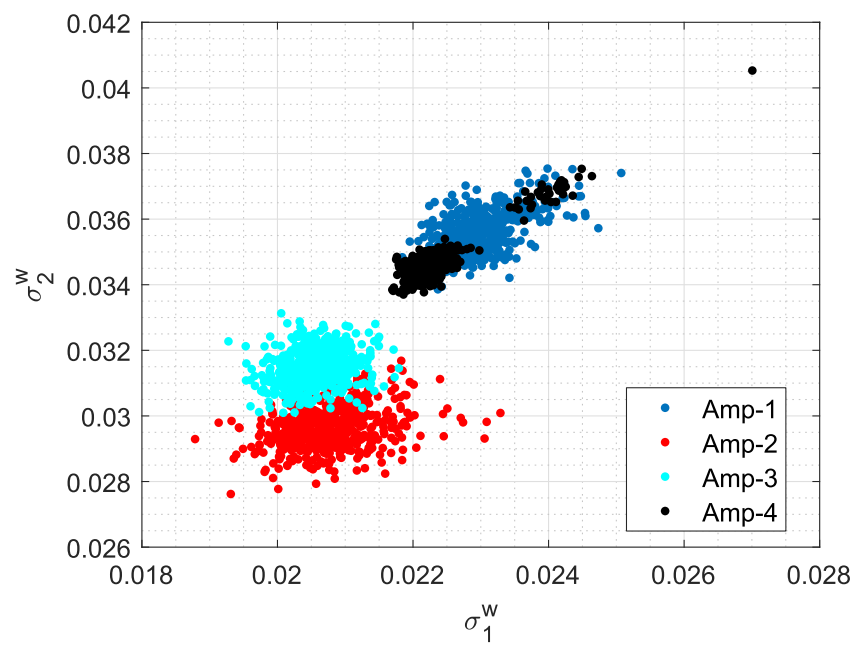

Fig. 15. Frequency standard deviation feature.

of measurements were obtained from the same solid state amplifiers whose data were analyzed in the previous section. The amplifiers were in the saturation regime and the data is obtained in a high SNR (SNR $\geq 50 \mathrm{~dB}$ ) regime. For illustrative purposes, a set of measurements is shown in Fig. 11. The part of the signal where UMOP occurs is extracted by using the same time window shown in Fig. 7. Then, extracted signal is fed into the VMD feature extraction stage where the features given in Table.I are obtained. Features obtained from the envelope of the radar signals are shown in Fig. 12, Fig. 14, and Fig. 15. As stated in [2], features should form natural clusters in the feature space for reliable classification. From the results we conclude that different emitters occupy separate regions of the feature space, and the amplifiers can be classified accurately by using a basic linear classifier.

In the second dataset, measurements obtained from an ELINT system operating in the field were used. The data set consist of 47 emitters. Some of these emitters were productions of the same radar. For each radar, we randomly generated 10 measurement sets of recorded pulses, where 


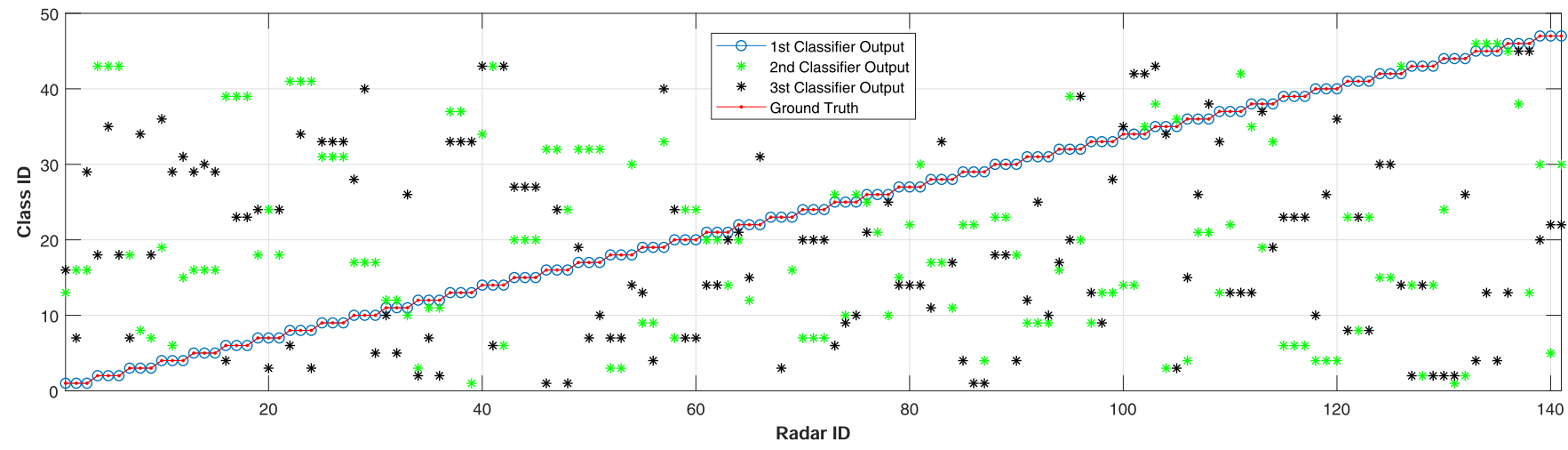

Fig. 16. Classification performance for 47 different radars.

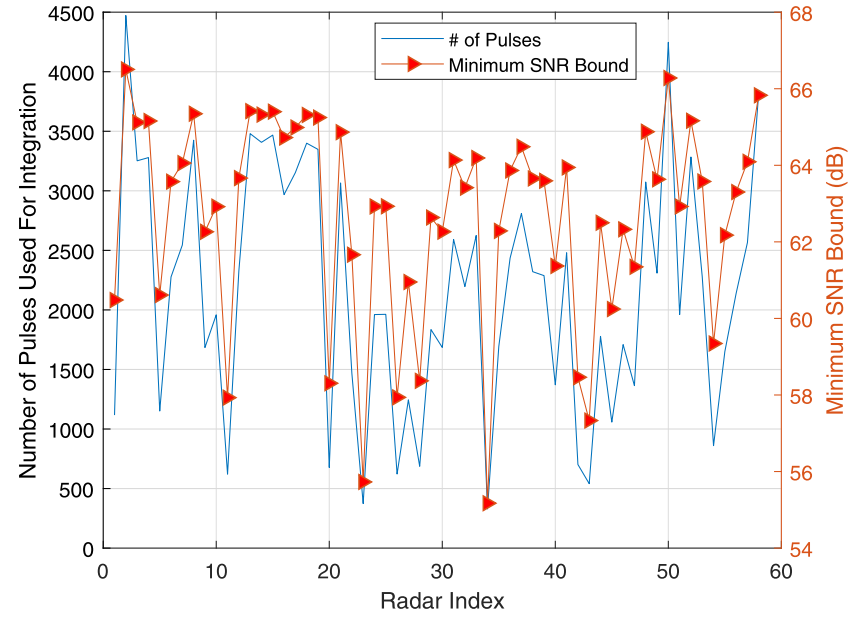

Fig. 17. Number of pulses used during data integration for each radar, and the corresponding SNR lower bound.

each set contains 200 pulses to be aligned and integrated. As a result, $47 \times 10=470$ different measurement sets are obtained. The Normalized Euclidean Distance of the extracted features between each measurement is plotted in Fig. 13 on a logarithmic scale. In this figure, every 10 by 10 sub-matrix around the diagonal belongs to the same class. It can be observed that in class distances are below $-50 \mathrm{~dB}$ whereas distance between classes are above $-20 \mathrm{~dB}$.

The classification performance of the proposed features is evaluated using a simple majority voting classifier [26], since we are mainly interested in the quality of the generated features. During the classification process, the class index whose corresponding feature has the closest distance is found from the database for each computed feature. Once this operation is utilized for all extracted features, the most frequently appearing class index is selected as the classification result. The second and third possible candidate classes are obtained similarly. Although prior information oo radars that can be obtained from classical parameters such as PW, PRI and RF can be used by practical SEI systems, these parameters are not used by the classifier. During the experiments, it is only assumed that the test data of the classifier belongs to one of the emitters in the database, i.e. it is not a new emitter.
During the classification experiments, we randomly generate 4 batches where each batch contains 400 pulses from 47 radars. As this dataset includes measurements of the same emitter that are obtained at different times over several years, we make sure that each batch consists of measurements that are taken at the same time. For each batch, we coherently integrate the pulses to obtain a high SNR measurement. One of these four signals for each radar is placed in a database as prior information and 3 other pulses are used for testing purposes. We therefore performed $47 \times 3=141$ classification tests. Obtained classification results are shown in Fig. 16. Our results show that the proposed technique can obtain $100 \%$ accuracy in this dataset.

Finally, we evaluate the performance of the SEI algorithm for different SNR levels and compare it with the EM ${ }^{2}$ technique given in [11]. This analysis has a few very important aspects. First of all, it provides an opportunity to fairly compare our technique with the available methods in the literature using our real radar dataset. Another important points is that it enables us to determine the number of pulses to be integrated that is required for successful SEI classification. Moreover, important system level requirements, such as antenna gain, can be derived so that the designed SEI system can capture the data at a sufficiently high SNR for SEI purposes. Unfortunately, obtaining the classification performance at different SNR levels using real radar data is not straight forward, due to the fact that data acquisition over multiple radars is a long term process, during which we have no direct control over the SNR of the received signal. But using our pulse alignment technique, we can generate measurements at a very high SNR (ideally at infinite SNR), and we can then obtain the SNR performance of our SEI technique by adding AWGN synthetically to test the performance at a desired SNR. To accomplish this task, we filtered the data so that each individual pulse had at least $30 \mathrm{~dB}$ SNR. During the SNR calculation, the pulse amplitude is calculated at the center of the pulse, so that the rise and fall times of the pulses which include some transient effects can be avoided. Assuming that we can perfectly align and integrate pulses, the effective SNR for $N$ pulses can be calculated in $\mathrm{dB}$ as:

$$
\mathrm{SNR}_{\min }=30+10 \log _{10} N
$$




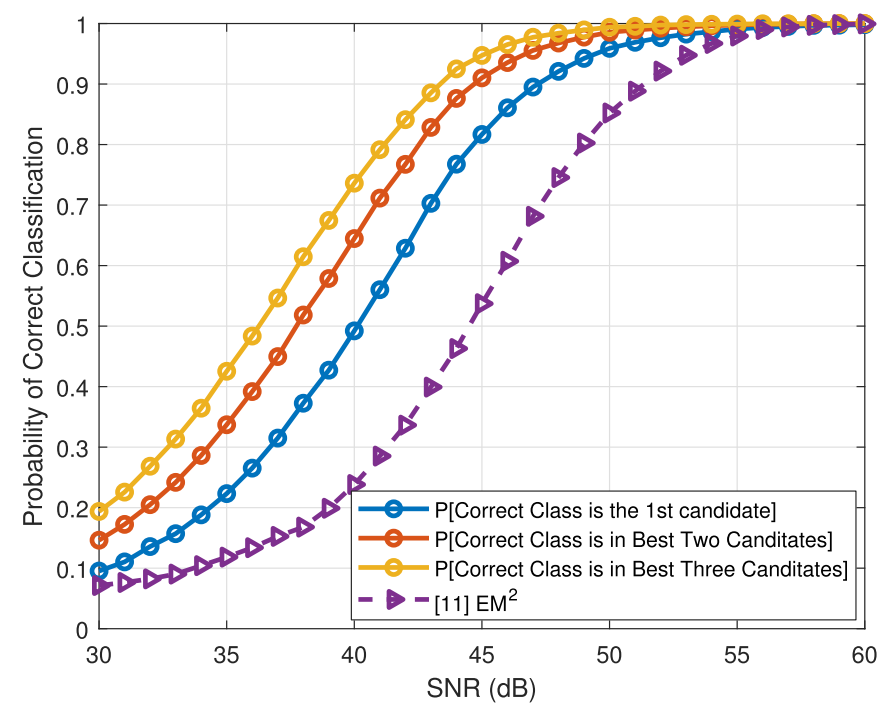

Fig. 18. Classification performance with respect to SNR.

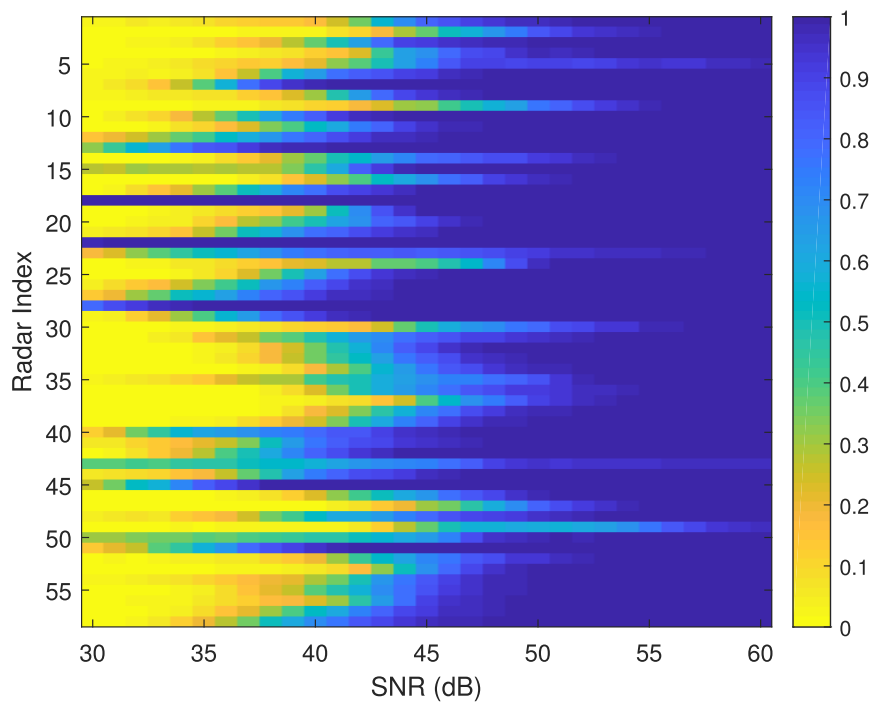

Fig. 19. Probability of correct classification of the proposed technique for each radar with respect to SNR.

In Fig. 17, the number of pulses for each radar and the corresponding effective SNR bounds are shown. Note that this bound shows the minimum SNR level, assuming that the SNR of each pulse is at least $30 \mathrm{~dB}$. As there are pulses with higher SNR, effective SNR for each radar is actually greater than the given bound. Also note that, the SNR calculation is conducted at the center of the pulse where no transient effects are present. As a result, the proposed SEI technique is actually working with samples that have much lower SNR which is different for each radar depending on their individual waveforms.

In Fig. 18, the overall classification performance that is obtained by using Monte Carlo simulations for SNR values between 30 to $60 \mathrm{~dB}$ is shown. Our results demonstrate that the effective SNR value should be around $47 \mathrm{~dB}$ to obtain a correct classification probability larger then 0.9. The performance of the proposed technique is also compared with the $\mathrm{EM}^{2}$ method given in [11]. It can be observed
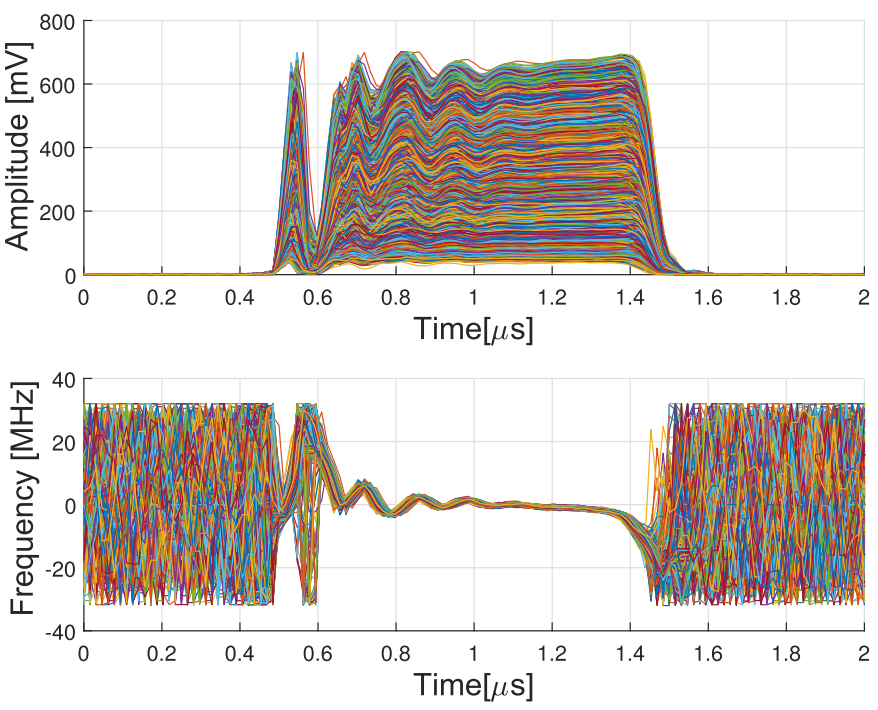

Fig. 20. Complex samples of radar index 22. (Amplitude on the top, instantaneous frequency on the bottom.)
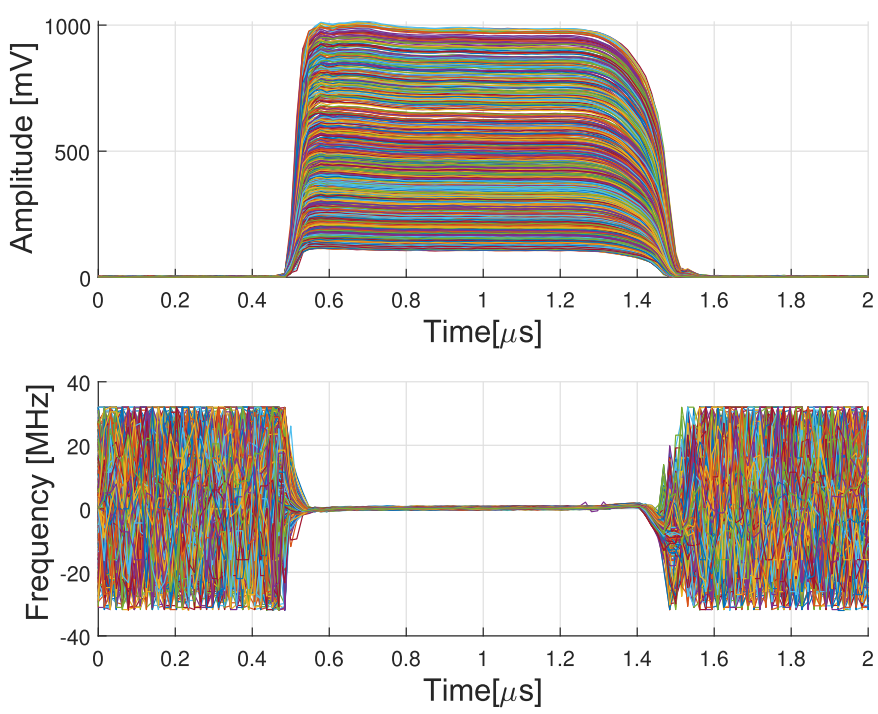

Fig. 21. Complex samples of radar index 49. (Amplitude on the top, instantaneous frequency on the bottom.)

that proposed technique outperforms $\mathrm{EM}^{2}$ and has an SNR advantage of $4 \mathrm{~dB}$ for a 0.9 probability of correct classification. Furthermore, considering that EW systems typically observe their targets at a SNR of around $30 \mathrm{~dB}$, we can conclude that we should integrate at least 100 pulses to achieve this level of performance. In Fig. 19, the SNR performance of each radar in the dataset is shown. It can be observed that classification performance is highly dependent on the radar type. For example, radar index 22 is easily distinguished from other emitters, whereas radar index 49 has a slightly lower classification performance compared to other radars. In Fig. 20, and Fig. 21, the envelopes of radar number 22 and 49 are given respectively. We can see that UMOP on radar 22 is very distinct, whereas the envelope of radar 49 is almost identical to an ideal square wave which is the main reason for the performance difference between these two emitters. 


\section{CONCLUSION}

In this work, we proposed a novel scheme for radar SEI. The proposed technique accurately perform time alignment and coherent integration of the collected radar pulses to boost the SNR so that UMOP on the signals can be reliably identified. Then, features related to UMOP are extracted using a VMDbased technique. The proposed technique is demonstrated successfully on real radar data sets that are significantly larger and more challenging than previously reported SEI results in the literature. Simulation results show the that proposed technique outperforms the state of the art $\mathrm{EM}^{2}$ method.

\section{REFERENCES}

[1] R. G. Wiley, ELINT: The Interception and Analysis of Radar Signals. Norwood, MA, USA: Artech House, 2006.

[2] K. I. Talbot, P. R. Duley, and M. H. Hyatt, "Specific emitter identification and verification," Technol. Rev., vol. 113, pp. 113-133, Jan. 2003.

[3] T.-w. Chen, W.-d. Jin, and J. Li, "Feature extraction using surroundingline integral bispectrum for radar emitter signal," in Proc. IEEE Int. Joint Conf. Neural Netw. (IEEE World Congr. Comput. Intelligence), Jun. 2008, pp. 294-298.

[4] B. Pei, Z. Bao, and M. Xing, "Logarithm bispectrum-based approach to radar range profile for automatic target recognition," Pattern Recognit. vol. 35, no. 11, pp. 2643-2651, Nov. 2002.

[5] M.-W. Liu and J. F. Doherty, "Specific emitter identification using nonlinear device estimation," in Proc. IEEE Sarnoff Symp., Apr. 2008, pp. $1-5$.

[6] A. Aubry, A. Bazzoni, V. Carotenuto, A. De Maio, and P. Failla, "Cumulants-based radar specific emitter identification," in Proc. IEEE Int. Workshop Inf. Forensics Secur., Nov. 2011, pp. 1-6.

[7] S. D'Agostino, G. Foglia, and D. Pistoia, "Specific emitter identification: Analysis on real radar signal data," in Proc. Eur. Radar Conf. (EuRAD), Oct. 2009, pp. 242-245.

[8] N. E. Huang et al., "The empirical mode decomposition and the Hilbert spectrum for nonlinear and non-stationary time series analysis," Proc. Roy. Soc. London. A, Math., Phys. Eng. Sci., vol. 454, no. 1971, pp. 903-995, Mar. 1998.

[9] C. Song, J. Xu, and Y. Zhan, "A method for specific emitter identification based on empirical mode decomposition," in Proc. IEEE Int. Conf. Wireless Commun., Netw. Inf. Secur., Jun. 2010, pp. 54-57.

[10] A. C. Polak and D. L. Goeckel, "Wireless device identification based on RF oscillator imperfections," IEEE Trans. Inf. Forensics Security, vol. 10, no. 12, pp. 2492-2501, Dec. 2015.

[11] J. Zhang, F. Wang, O. A. Dobre, and Z. Zhong, "Specific emitter identification via Hilbert-Huang transform in single-hop and relaying scenarios," IEEE Trans. Inf. Forensics Security, vol. 11, no. 6, pp. 1192-1205, Jun. 2016.

[12] G. Huang, Y. Yuan, X. Wang, and Z. Huang, "Specific emitter identification based on nonlinear dynamical characteristics," Can. J. Electr. Comput. Eng., vol. 39, no. 1, pp. 34-41, 2016.

[13] J. Zhang, F. Wang, Z. Zhong, and O. Dobre, "Novel Hilbert spectrumbased specific emitter identification for single-hop and relaying scenarios," in Proc. IEEE Global Commun. Conf. (GLOBECOM), Dec. 2015, pp. 1-6.

[14] U. Satija, N. Trivedi, G. Biswal, and B. Ramkumar, "Specific emitter identification based on variational mode decomposition and spectral features in single hop and relaying scenarios," IEEE Trans. Inf. Forensics Security, vol. 14, no. 3, pp. 581-591, Mar. 2019.

[15] B. He, F. Wang, Y. Liu, and S. Wang, "Specific emitter identification via multiple distorted receivers," in Proc. IEEE Int. Conf. Commun. Workshops (ICC Workshops), May 2019, pp. 1-6.

[16] Y. Pan, S. Yang, H. Peng, T. Li, and W. Wang, "Specific emitter identification based on deep residual networks," IEEE Access, vol. 7, pp. 54425-54434, 2019.

[17] L. Ding, S. Wang, F. Wang, and W. Zhang, "Specific emitter identification via convolutional neural networks," IEEE Commun. Lett., vol. 22, no. 12, pp. 2591-2594, Dec. 2018.

[18] G. Gok, Y. K. Alp, and F. Altiparmak, "Radar fingerprint extraction via variational mode decomposition," in Proc. 25th Signal Process. Commun. Appl. Conf. (SIU), May 2017, pp. 1-4.
[19] R. H. Bartels, J. C. Beatty, and B. A. Barsky, "Hermite and cubic spline interpolation," in An Introduction to Splines for Use in Computer Graphics and Geometric Modeling. San Mateo, CA, USA: Morgan Kaufmann, 1998, pp. 9-17.

[20] K. Dragomiretskiy and D. Zosso, "Variational mode decomposition," IEEE Trans. Signal Process., vol. 62, no. 3, pp. 531-544, Feb. 2014.

[21] V. Valimaki and A. Haghparast, "Fractional delay filter design based on truncated Lagrange interpolation," IEEE Signal Process. Lett., vol. 14 no. 11, pp. 816-819, Nov. 2007.

[22] L. H. Thomas, "Elliptic problems in linear difference equations over a network," Watson Sci. Comput. Lab. Rep., Columbia Univ., New York, NY, USA, 1949, vol. 1.

[23] S. Stein, "Algorithms for ambiguity function processing," IEEE Trans. Acoust., Speech, Signal Process., vol. ASSP-29, no. 3, pp. 588-599, Jun. 1981.

[24] B. Boashash, Time-Frequency Signal Analysis and Processing: A Comprehensive Reference. New York, NY, USA: Academic, 2015.

[25] M. R. Hestenes, "Multiplier and gradient methods," J. Optim. Theory Appl., vol. 4, no. 5, pp. 303-320, Nov. 1969.

[26] L. Lam and S. Y. Suen, "Application of majority voting to pattern recognition: An analysis of its behavior and performance," IEEE Trans. Syst., Man, Cybern. A, Syst., Humans, vol. 27, no. 5, pp. 553-568, Sep. 1997.

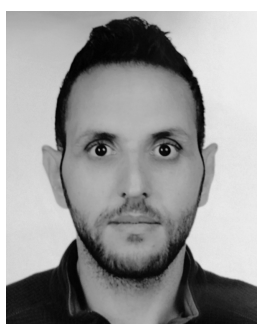

Gokhan Gok was born in Ankara, Turkey, in 1987. He received the B.Sc. degree in electrical and electronics engineering from Hacettepe University, Ankara, in 2010, and the M.S. degree in electrical and computer engineering from Middle East Technical University, Ankara, in 2013. He is currently pursuing the Ph.D. degree with Bilkent University, Ankara. He is also working as a Senior Algorithm Design Engineer at the Radar, Electronic, Warfare and Intelligence Systems Division, ASELSAN Inc. His research interests include digital signal processing, array signal processing, sensor calibration, and remote sensing.

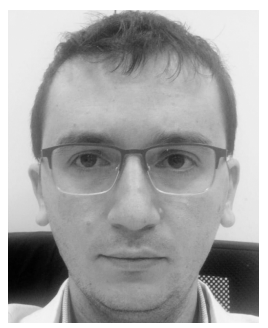

Yasar Kemal Alp was born in Konya, Turkey, in 1985. He received the B.Sc. and Ph.D. degrees in electrical and electronics engineering from Bilkent University, Ankara, Turkey, in 2007 and 2012, respectively. He worked as a Research Scientist in Schlumberger Cambridge Research from June August 2009 to July September 2010. He is currently working as the Lead Algorithm Design Engineer with the Radar, Electronic Warfare and Intelligence Systems Division, ASELSAN Inc. His research interests are digital signal processing, timefrequency analysis, and inverse problems and their applications to radar signal processing.

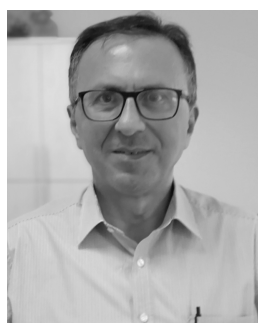

Orhan Arikan (Member, IEEE) was born in Manisa, Turkey, in 1964. He received the B.Sc. degree in electrical and electronics engineering from Middle East Technical University, Ankara, Turkey, in 1986, and the M.S. and Ph.D. degrees in electrical and computer engineering from the University of Illinois at Urbana-Champaign in 1988 and 1990, respectively. Following his graduate studies, he was employed as a Research Scientist with the Schlumberger-Doll Research Center, Ridgefield, CT, USA. In 1993, he joined the Electrical and Electronics Engineering Department, Bilkent University, Ankara. Since 2006, he has been a Full Professor with Bilkent University. He has served as the Chairman of the Department from 2011 to 2019. His current research interests include statistical signal processing, time-frequency analysis, and remote sensing. He has also served as the Chairman of the Turkey Chapter of IEEE. 